\title{
Properties of the SX Phœnecis Stars in the Metal-Poor Globular Cluster NGC 5053, and Pulsation Modes of Three of the Newly-Discovered SX Phe Stars in 47 Tuc $^{1}$
}

\author{
James M. Nemec \\ Department of Physics \& Astronomy, York University, ON Canada
}

\begin{abstract}
The properties of variable blue stragglers (i.e., SX Phe stars) in the extremely metal-poor globular cluster NGC 5053 are reviewed. Pulsation modes are derived for three of the SX Phe stars recently discovered in the central regions of 47 Tuc by Gilliland et al. (1995a,b).
\end{abstract}

\section{Properties of the SX Phœenecis stars in NGC 5053}

Nemec et al. (1995b) have recently monitored for variability 16 of the 28 presentlyknown blue stragglers (BSs) in the very metal-poor globular cluster NGC 5053, and identified five of the 16 stars as SX Phe stars. As a group the five stars have the shortest periods (49<P<57 min), lowest luminosities $\left(3.0 \leq M_{V} \leq 3.3\right.$ mag) and smallest amplitudes $\left(0.08 \leq A_{V} \leq 0.25 \mathrm{mag}\right)$ of the SX Phe stars that are presently known. According to their colours and locations in both $B$ and $V$ period-luminosity diagrams, four of the five SX Phe stars (Nos. 11, 13, 14 and 15 in the Nemec \& Cohen 1989 catalog) appear to be fundamental-mode pulsators, while the fifth and bluest star, No. 7, appears to be a first-overtone pulsator. One of the stars, No. 11, exhibits cycle-to-cycle light variations that are suggestive of multi-mode pulsations (unfortunately, not enough photometry is available to derive the secondary pulsation period). Estimated masses for the stars, derived both directly from the pulsation equation and indirectly by comparison with the RR Lyr stars, range from $0.53 M_{\odot}$ for the candidate first-overtone star $\mathrm{NC} 7$, to $1.8 M_{\odot}$ for $\mathrm{NC} 13$. While the latter is comparable with twice the main sequence turnoff mass, the very low mass for NC7 is surprising unless the derived colour is too blue (additional colour observations are needed to rule out this possibility). Because of their short periods the NGC 5053 SX Phe stars are important for establishing period-luminosity-metallicity relations. Based on the new photometry of the SX Phe stars, and contemporaneous photometry of the RR Lyrae stars (Nemec, Mateo \& Schombert 1995a), the estimated slopes of the $P-L-[\mathrm{Fe} / \mathrm{H}]$ relations are $-2.16 \pm 0.46(B)$ and $-2.13 \pm 0.46(V)$, with assumed metallicity coefficients $c_{B}=0.35, c_{V}=0.32$, and extinction coefficients $R_{B}=0.41$ and $R_{V}=3.1$ (see Nemec, Linnell Nemec \& Lutz 1994). Assuming $E_{\mathrm{B}-\mathrm{V}}=0.06$, the estimated true distance modulus of NGC 5053 is $(m-M)_{0}=16.06$.

\footnotetext{
${ }^{1}$ Dedicated to the memory of Thomas E. Lutz and William H. Wehlau.
} 
In addition to the SX Phe stars, several non-variable NGC 5053 stars were found inside the Pop. II instability strip. These may be the result of rapid rotation which prevents the stars from pulsating. Measurements of the rotation velocities of the NGC 5053 blue stragglers will be required to test this idea.

\section{Pulsation modes of three SX Phe stars in the core of 47 Tuc}

At this conference Gilliland et al. (1995a) announced the exciting discovery of three SX Phe stars in the central region of the metal-rich globular cluster 47 Tuc. These stars, with their high metallicities, should prove useful in the investigation of possible dependencies of the $P-L$ relations on $[\mathrm{Fe} / \mathrm{H}]$. One of the stars is doubly-periodic with pulsation periods of 2.447 hours and 1.890 hours (see also Gilliland et al. 1995b), the ratio of which, $P_{1} / P_{0}=0.772 \pm 0.001$, is similar to that of SX Phe itself and is indicative of simultaneous pulsation in the fundamental and first-overtone modes. The other two stars have pulsation periods of 91.07 min and $80.20 \mathrm{~min}$, and mean $V$ magnitudes of 15.3 and 15.8 mag, respectively. The mean period of the 47 Tuc SX Phe stars is much longer than that of the NGC 5053 SX Phe stars, suggesting a possible dependence of pulsation period on $[\mathrm{Fe} / \mathrm{H}]$. The stars also extend the $P-L$ relations to longer periods than previously available. Comparison of the mean $V$ magnitudes and periods to the period-adjusted-magnitude relations for SX Phe stars (Nemec et al. 1995b) demonstrates that all three 47 Tuc stars lie on or near those lines, and suggests that the 80 -min star is a fundamental-mode pulsator and that the 91-min star is a first-overtone star. A preliminary analysis suggests that with the inclusion of the 47 Tuc stars the slope of the $V$ relation increases from $-2.13 \pm 0.46$ (Nemec et al. 1995b) to $-2.28 \pm 0.39$. On the other hand, the derived distance modulus for 47 Tuc appears to be $\sim 0.7$ mag smaller than previous estimates, indicating that either the preliminary mean $V$ magnitudes are too bright or the assumed metallicity dependence is in error. Final reductions of the 47 Tuc HST data are eagerly awaited.

The author thanks his collaborators, and Michael Breger, Alistair Walker and Doug Welch for questions that helped improve this paper.

\section{References}

Gilliland, R.L., Edmonds, P.D., Petro, L. Saha, A. \& Shara, M.M. 1995a, poster paper, these proceedings.

Gilliland, R.L., Edmonds, P.D., Petro, L. Saha, A. \& Shara, M.M. 1995b, ApJ, STScI Preprint No. 909.

Nemec, J.M. \& Cohen, J.G. 1989, ApJ, 336, 780

Nemec, J.M., Linnell Nemec, A.F. \& Lutz, T. 1994, AJ, 108, 222

Nemec, J.M., Mateo, M. \& Schombert, J.M. 1995a, AJ, 109, 618

Nemec, J.M., Mateo, M., Burke, M. \& Olszewski, E.W. 1995b, AJ, in press 